\title{
Manoeuvring-based actuation evaluation of an AUV with control surfaces and through-body thrusters
}

\author{
Xianbo Xiang, ${ }^{\mathrm{a}, \mathrm{b}}$, Caoyang Yuc,*, Qin Zhang ${ }^{\mathrm{d}}$, Philip A. Wilson ${ }^{\mathrm{e}}$, \\ Guohua $\mathrm{Xu}^{\mathrm{a}}$ \\ ${ }^{a}$ School of Naval Architecture and Ocean Engineering, Huazhong University of Science \\ and Technology, Wuhan 430074, China \\ ${ }^{b}$ Shenzhen Huazhong University of Science and Technology Research Institute, Shenzhen \\ 518057, China \\ ${ }^{c}$ School of Oceanography, Shanghai Jiao Tong University, Shanghai 200030, China \\ ${ }^{d}$ State Key Lab of Digital Manufacturing, Equipment and Technology, Huazhong \\ University of Science and Technology, Wuhan 430074, China \\ ${ }^{e}$ Faculty of Engineering and Physical Sciences, University of Southampton, Southampton \\ SO16 7QF, United Kingdom
}

\begin{abstract}
For an autonomous underwater vehicle (AUV), control surfaces are usually used for high-speed manoeuvres, while at low speed the AUV relies upon through-body thrusters for manoeuvring. In this paper, the speed intervals of high-speed and low-speed manoeuvres are quantitatively analyzed. To this end, the AUV's dynamics equations composed of body hydrodynamics and actuator models are first established with specific regard to operation at different speeds. Resorting to the above complete dynamics model, turning manoeuvring tests are carried out to forecast the AUV's advance and turning time at different speeds. Initial turning ability criterion approved by International Maritime Organization is then used to calculate thresholds for low-speed and high-speed manoeuvres. Due to non-overlap, the entire speed profile is divided into low-speed, medium-speed and high-speed intervals, where different actuator combinations and characteristics are analyzed. Finally, a smooth switching law is designed to manage the contribution of two types of actuators through the entire AUV's speed profile.
\end{abstract}

Keywords:

\footnotetext{
${ }^{*}$ Corresponding author

Email address: yucaoyang@sjtu.edu.cn (Caoyang Yu)
} 
Autonomous underwater vehicles, manoeuvring test, actuation evaluation, unified control.

\section{Introduction}

With the development of control, communication and sensory technologies, the use of underwater vehicles including remotely operated vehicles (ROVs), autonomous underwater vehicles (AUVs) and autonomous underwater gliders (AUGs), has increased rapidly to perform complex and highrisk underwater missions. Usually the sensory technology is relied on to make decisions regarding underwater mission, and then the manoeuvres are performed to complete the mission with the aid of a high-performance control system $[1,2,3]$.

Recently much attention has been paid to the development of such an onboard system to allow an AUV to manoeuvre with similar capability and precision as a ROV but maintain a long endurance that is typical of a normal AUV. Applications for such a vehicle include search and inspection of underwater features of interest such as subsea pipelines, hydrothermal vents, and underwater mines $[4,5,6]$. To achieve this goal, the AUV must be able to hover (maintain depth/heading at zero speed) as well as fly at high speed to cover a large area. Vehicles have been built in the past that offer this capability, such as Delphin2, SeaCat, Sparus II and Yangji [7, 8, 9, 10]. As shown in Fig. 1, this type of vehicles usually has a slender body that is equipped with through-body thrusters and control surfaces used for hover and flight, respectively.

The performance of actuators used must be known before controller design of an AUV. For high-speed flight control, stern control surfaces are the key factors. In [11], a six-degrees-of-freedom nonlinear dynamics model and performance prediction for the WL-II AUV with control surfaces were investigated where hydrodynamic coefficients were obtained by Computational Fluid Dynamics (CFD) method. The input saturation of actuators was investigated in $[12,13]$. Based on the high-speed nonlinear dynamics model, plenty of advanced controllers including sliding mode control, fuzzy logic control and neural network control are designed for various control scenarios, such as path following, trajectory tracking and path tracking $[14,15,16,17]$. It is noted that the vehicle is usually under-actuated when operating at high speeds. 


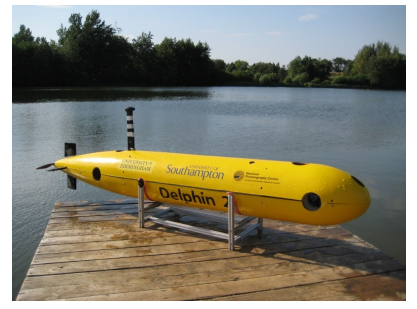

(a) Delphin2

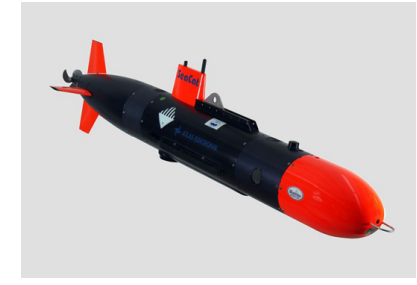

(b) SeaCat

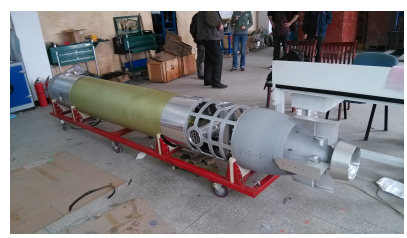

(c) Yangji

Figure 1: AUVs capable of hover and flight

For low-speed motion including hover mode, through-body thrusters should be used because at this time the effective torque of control surfaces is relatively small. In [18], it was concluded that a function that incorporated both depth and thruster speed must be derived to estimate the total thrust whilst hovering, because it is significantly reduced as the thruster is near the free surface. The work in [7] illustrated changes in magnitude and location of pressure across the vehicle hull will cause a reduction in effective thrust of through-body thrusters when the surge speed is non-zero. The thrust acting on the vessel depending on the vehicle forward speed was proposed in [19] and then a piecewise nonlinear model that also considers the heading angle was discussed in [20]. The complete dynamics modelling and hover style operation of the Delphin2 AUV including through-body thrusters were verified by simulation and experimental results, as presented in [21]. Yet, the preceding literature review has not yet given accurate low-speed and high-speed intervals for an AUV capable of hover and flight by considering the effects of both control surfaces and through-body thrusters.

This paper first builds the complete motion model of the Yangji AU$\mathrm{V}$ based on the previous results, especially the through-body Model-280 thrusters. Another aim of this paper is to seek accurate intervals of low speed and high speed for the Yangji AUV developed at the lab of autonomous robotic marine system (ARMS), which aims to perform environmental survey 
and monitoring tasks in medium-range shallow water. The next two sections are complete motion modelling and actuation evaluation based on manoeuvring tests, respectively. Finally, conclusion and future work are summarized in section 4 .

\section{Complete AUV motion modelling}

\subsection{Yangji AUV description}

As shown in Fig. 1(c), the Yangji AUV shows a torpedo-like shape and is divided into the bow, middle and stern parts. It is $5.3 \mathrm{~m}$ long with a maximum diameter of $0.62 \mathrm{~m}$, weighs $381 \mathrm{~kg}$ in air, and can dive to the depth up to $300 \mathrm{~m}$. Obviously, the vehicle is equipped with four throughbody thrusters, two for horizontal motion and the others for vertical motion, and two pairs of stern control surfaces driven by rudders. The distances form the center of gravity of the Yangji AUV to the center of the bow horizontal through-body thruster and to the stern thruster are $1.48 \mathrm{~m}$ and $1.64 \mathrm{~m}$, respectively. The area of each stern control surface is approximately $0.02 \mathrm{~m}^{2}$ and its aspect ratio is designed as 1. In addition, the diameter of throughbody thrusters is $0.117 \mathrm{~m}$ and the corresponding channel increases by 0.021 $\mathrm{m}$.

\subsection{Kinematics and dynamics modelling}

For slender bodies (large length/width ration), it is a common assumption that the six degrees-of-freedom equations of motion are divided into two non-interacting (or lightly interacting) subsystems: longitudinal subsystem and lateral subsystem [22]. As illustrated in Fig. 2, in order to explicitly describe the Yangji AUV operated in horizontal plane, the following standard coordinate frames and notations are first defined. Let $\{I\}$ be the inertial frame and $\{B\}$ be the body frame. The posture vector of the AUV $\boldsymbol{\eta}=$ $[x, y, \psi]^{T}$ is described in frame $\{I\}$, where $x$ and $y$ denote the horizontal position, and $\psi$ is the heading angle. The velocity vector $\boldsymbol{\nu}=[u, v, r]^{T}$ is described in frame $\{B\}$, where $u, v$ and $r$ are the surge, sway and yaw speeds, respectively.

The horizontal-plane kinematics equations of the AUV are usually described as follows [23]:

$$
\dot{\boldsymbol{\eta}}=\boldsymbol{J}(\psi) \boldsymbol{\nu}
$$




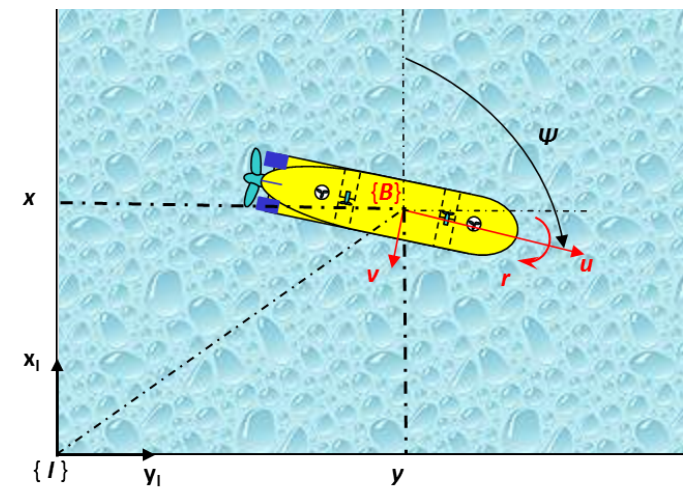

Figure 2: AUV reference frames

where $\boldsymbol{J}(\psi)=\left(\begin{array}{ccc}\cos (\psi) & -\sin (\psi) & 0 \\ \sin (\psi) & \cos (\psi) & 0 \\ 0 & 0 & 1\end{array}\right)$ is the Euler rotation matrix from frame $\{B\}$ to frame $\{I\}$. Thus, Eq. (1) can be expanded as

$$
\left\{\begin{aligned}
\dot{x} & =u \cos (\psi)-v \sin (\psi) \\
\dot{y} & =u \sin (\psi)+v \cos (\psi) \\
\dot{\psi} & =r
\end{aligned}\right.
$$

For the horizontal-plane AUV operation, roll and pitch angles are assumed to be negligibly small; hence, the vector of gravitational forces and moments can be omitted. Let $\boldsymbol{M}, \boldsymbol{C}(\boldsymbol{\nu}), \boldsymbol{D}(\boldsymbol{\nu})$ and $\boldsymbol{\tau}$ be the inertia matrix, Coriolis and centripetal matrix, damping matrix and vector of control inputs, respectively. In the absence of environmental disturbances, the horizontal-plane dynamics equations of the AUV are then described as follows:

$$
M \dot{\nu}=-C(\nu) \nu-D(\nu) \nu+\tau
$$

According to [24], the simplified horizontal-plane dynamics model of the 
Yangji AUV in (3) can be expanded as

$$
\left\{\begin{aligned}
\left(m-X_{\dot{u}}\right) \dot{u}= & m v r+m x_{g} r^{2}-Y_{\dot{v}} v r+X_{|u| u}|u| u \\
& +\rho D^{4} K_{T}\left(1-t_{T}\right) n|n| \\
\left(m-Y_{\dot{v}}\right) \dot{v}+m x_{g} \dot{r}= & \left(X_{\dot{u}}-m\right) u r+Y_{v} v+Y_{r} r+Y_{|v| v \mid}|v| v+Y_{r|r|} r|r| \\
& +f\left(U_{h 1}\right)+f\left(U_{h 2}\right) \\
\left(I_{z}-N_{\dot{r}}\right) \dot{r}+m x_{g} \dot{v}= & -m x_{g} u r+Y_{\dot{v}} u v-X_{\dot{u}} u v \\
& +N_{r} r+N_{v} v+N_{|r| r}|r| r+N_{|v| v}|v| v \\
& +f\left(U_{h 1}\right) l_{h 1}+f\left(U_{h 2}\right) l_{h 2}+N_{\delta r} \delta_{r}
\end{aligned}\right.
$$

where $m$ is the AUV's mass, $I_{z}$ is the moment of inertia about $z$-axis of fame $\{B\}$ and $x_{g}$ is the abscissa of the AUV's gravity in frame $\{B\}$. In addition, $X_{(\cdot)}, Y_{(\cdot)}$ and $N_{(\cdot)}$ denote the AUV's hydrodynamics coefficients. For the stern propeller, $\rho$ is water density, $D$ is diameter, $K_{T}$ is thrust coefficient, $t_{T}$ is thrust deduction factor, and $n$ is propeller speed, measured in rpm. $f(U)$ and $l_{(\cdot)}$ denote the thrust generated by through-body thrusters when input voltage is equal to $U$ and the corresponding arm of force, respectively. $\delta_{r}$ is the angle of vertical control surfaces.

Remark 1. For the Yangji AUV, $m=381 \mathrm{~kg}, x_{g}=0 \mathrm{~m}, I_{z}=2131.29$ $\mathrm{kg} \cdot \mathrm{m}^{2}, \rho D^{4} K_{T}\left(1-t_{T}\right)=1.09 \times 10^{-4} \mathrm{~kg} \cdot \mathrm{m}, l_{h 1}=1.48 \mathrm{~m}$ and $l_{h 2}=1.64$ m. Up to now, four commonly-used methods including captive model test [25], empirical formula estimation [26], computational fluid dynamics [27] and system identification [28] are resorted to obtain dynamics model. Based on captive model test and empirical formula estimation, the nominal dynamics coefficients of the Yangji AUV except through-body thrusters are derived, as listed in Table 1, where $X_{(\cdot)}^{\prime}$ denotes the dimensionless coefficient of $X_{(\cdot)}$.

\subsection{Through-body thruster modelling}

For through-body thrusters of the Yangji AUV, because their control input is voltage instead of propeller speed, we cannot adopt standard propeller theory. On the other hand, when a through-body tunnel thruster is operating, it sucks water into one side of the tunnel and ejects a jet of water on the opposite side, as demonstrated in Fig. 3. This water jet can be considered as an increase in the effective frontal area of the AUV, increasing the vehicle drag. As the AUV's speed increases, this effect is most pronounced and the thrust efficiency reduces substantially.

To model through-body thrusters, their thrusts against surge speed $u$ are measured by the team of ship manoeuvring and control laboratory in 
Table 1: Hydrodynamics coefficients

\begin{tabular}{cccc}
\hline Dimensionless & Value & Parameter & Unit \\
\hline$X_{\dot{u}}^{\prime}$ & $-2.681 \times 10^{-3}$ & $X_{\dot{u}}=\frac{1}{2} \rho L^{3} X_{\dot{u}}^{\prime}$ & $\mathrm{kg}$ \\
$X_{|u| u}^{\prime}$ & $-1.573 \times 10^{-3}$ & $X_{|u| u}=\frac{1}{2} \rho L^{2} X_{|u| u}^{\prime}$ & $\mathrm{kg} / \mathrm{m}$ \\
$Y_{\dot{v}}^{\prime}$ & $-1.998 \times 10^{-2}$ & $Y_{\dot{v}}=\frac{1}{2} \rho L^{3} Y_{\dot{v}}^{\prime}$ & $\mathrm{kg}$ \\
$Y_{v}^{\prime}$ & $-1.084 \times 10^{-2}$ & $Y_{v}=\frac{1}{2} \rho L^{2} u Y_{v}^{\prime}$ & $\mathrm{kg} / \mathrm{s}$ \\
$Y_{r}^{\prime}$ & $9.979 \times 10^{-3}$ & $Y_{r}=\frac{1}{2} \rho L^{3} u Y_{r}^{\prime}$ & $\mathrm{kg} / \mathrm{s}$ \\
$Y_{|v| v}^{\prime}$ & $-1.149 \times 10^{-1}$ & $Y_{|v| v}=\frac{1}{2} \rho L^{2} Y_{|v| v}^{\prime}$ & $\mathrm{kg} / \mathrm{s}$ \\
$Y_{r|r|}^{\prime}$ & $-7.725 \times 10^{-3}$ & $Y_{|r| r}=\frac{1}{2} \rho L^{4} Y_{|r| r}^{\prime}$ & $\mathrm{kg} \cdot \mathrm{m} / \mathrm{rad} / \mathrm{s}$ \\
$N_{\dot{r}}^{\prime}$ & $-1.338 \times 10^{-3}$ & $N_{\dot{r}}=\frac{1}{2} \rho L^{5} N_{\dot{r}}^{\prime}$ & $\mathrm{kg} \cdot \mathrm{m}^{2} / \mathrm{rad}$ \\
$N_{r}^{\prime}$ & $-4.813 \times 10^{-3}$ & $N_{r}=\frac{1}{2} \rho L^{4} u N_{r}^{\prime}$ & $\mathrm{kg} \cdot \mathrm{m}^{2} / \mathrm{rad} / \mathrm{s}$ \\
$N_{v}^{\prime}$ & $-1.071 \times 10^{-2}$ & $N_{v}=\frac{1}{2} \rho L^{3} u N_{v}^{\prime}$ & $\mathrm{kg} \cdot \mathrm{m} / \mathrm{s}$ \\
$N_{|r| r}^{\prime}$ & $-5.054 \times 10^{-3}$ & $N_{|r| r}=\frac{1}{2} \rho L^{5} N_{|r| r}^{\prime}$ & $\mathrm{kg} \cdot \mathrm{m}^{2} / \mathrm{rad}{ }^{2}$ \\
$N_{|v| v}^{\prime}$ & $1.805 \times 10^{-2}$ & $N_{|v| v}=\frac{1}{2} \rho L^{3} N_{|v| v}^{\prime}$ & $\mathrm{kg}$ \\
$N_{\delta r}^{\prime}$ & $1.74 \times 10^{-3}$ & $N_{\delta r}=\frac{1}{2} \rho L^{3} u^{2} N_{\delta r}^{\prime}$ & $\mathrm{kg} \cdot \mathrm{m}^{2} / \mathrm{rad}$ \\
\hline
\end{tabular}

the towing tank of Shanghai Jiao Tong University, and the corresponding results are listed in Table 2 , in which the thrusts corresponding to low voltage inputs are omitted when the speed is faster than $1 \mathrm{kn}$ because they are too small. It is obvious that the efficient thrust generated by through-body thrusters reduces substantially when the surge speed increases; for instance, the experiment value in voltage of $4 \mathrm{~V}$ is $17.464 \mathrm{~N}, 15.606 \mathrm{~N}, 9.805 \mathrm{~N}, 5.096$ $\mathrm{N}, 2.751 \mathrm{~N}$ when periodically increasing the surge speed of $0.5 \mathrm{kn}$.

In this paper, the through-body thruster's modelling is divided into two steps: Step one is static thrust and step two is considering deduction factors due to AUV's motion. First, choose the zero-speed group of data and fit static thrust with regard to voltage. Through the cftool toolbox of MATLAB, a polynomial $f(U)$ of degree 2 that is a best fit (in a least-squares sense) is chosen for experiment data and the corresponding mathematical expression is given by

$$
f\left(\left.U\right|_{u=0}\right)=\left\{\begin{array}{r}
1.592 U^{2}-2.102 U+0.495,|U|>0.7 \\
0, \text { otherwise }
\end{array}\right.
$$

where the dead-zone of through-body thrusters is within the region of $[-0.7$, $0.7] \mathrm{V}$. 


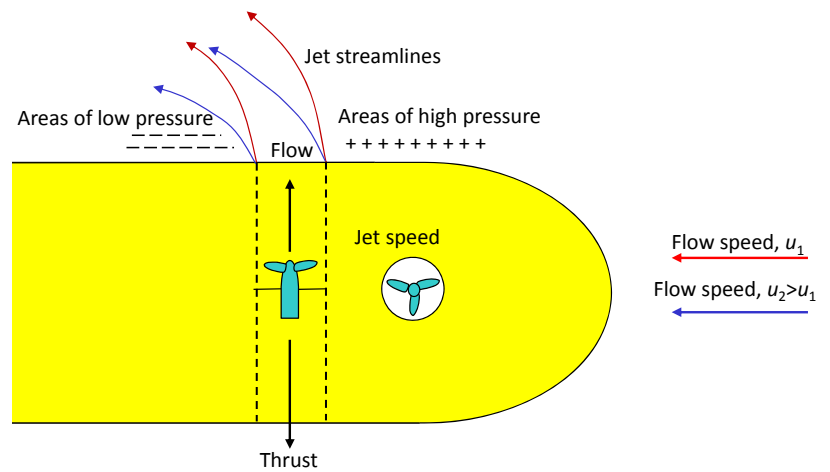

Figure 3: Influence of through-body thrusters at speed

Second, we choose five data of voltage $3.5 \mathrm{~V}$ and fit dynamic thrust with regard to surge speed, in order to derive deduction factors $1.078 e^{-1.654 u}$ due to AUV's motion. Combining these two steps, the thrust of a through-body thruster is concluded as follows:

$$
f(U)=\left\{\begin{array}{r}
\left(1.716 U^{2}-2.265|U|+0.533\right) e^{-1.654 u} \operatorname{sgn}(U),|U|>0.7 \\
0, \text { otherwise }
\end{array}\right.
$$

where the sign function $\operatorname{sgn}(U)$ is defined by

$$
\operatorname{sgn}(U)= \begin{cases}1, & U>0 \\ 0, & U=0 \\ -1, & U<0\end{cases}
$$

Comning Eqs. (6) and (7), we calculate 21 groups of fitting values and list them in the fourth column of Table 2. The corresponding root-mean-square error (RMSE) is $1.183 \mathrm{~N}$ according to

$$
R M S E=\sqrt{\frac{1}{21} \sum_{i=1}^{21}\left(f_{i}-e_{i}\right)^{2}}
$$

with $f_{i}$ being the experimental value and $e_{i}$ being the fitting value. It is noted that the RMSE is much smaller than the maximum value $17.464 \mathrm{~N}$ and the corresponding ratio is about 1:15 within an acceptable level. Combing RMSE and the comparison of the experiment and fitting curves illustrated in Fig. 4, it can be concluded that Eqs. (6) and (7) can well fit the thrust of through-body thrusters in the presence of surge motion. 
Table 2: Thrust performance of through-body thrusters

\begin{tabular}{cccc}
\hline Surge speed $/ \mathrm{kn}$ & Voltage/V & Experimental value/N & Fitting value/N \\
\hline 0.0 & 0.7 & 0.000 & -0.211 \\
0.0 & 1.5 & 0.757 & 0.996 \\
0.0 & 2.0 & 2.720 & 2.867 \\
0.0 & 2.5 & 4.564 & 5.596 \\
0.0 & 3.0 & 8.869 & 9.184 \\
0.0 & 3.5 & 12.763 & 13.630 \\
0.0 & 4.0 & 17.464 & 18.934 \\
0.5 & 2.0 & 1.242 & 1.874 \\
0.5 & 2.5 & 2.958 & 3.658 \\
0.5 & 3.0 & 6.489 & 6.004 \\
0.5 & 3.5 & 10.788 & 8.910 \\
0.5 & 4.0 & 15.606 & 12.378 \\
1.0 & 3.0 & 2.678 & 3.925 \\
1.0 & 3.5 & 6.018 & 5.825 \\
1.0 & 4.0 & 9.805 & 8.092 \\
1.5 & 3.0 & 1.035 & 2.566 \\
1.5 & 3.5 & 2.536 & 3.808 \\
1.5 & 4.0 & 5.096 & 5.290 \\
2.0 & 3.0 & 1.568 & 1.677 \\
2.0 & 3.5 & 2.048 & 2.490 \\
2.0 & 4.0 & 2.751 & 3.459 \\
\hline
\end{tabular}

\section{Manoeuvring forecast and actuation evaluation}

In this section, based on the complete motion equations of the Yangji AU$\mathrm{V}$ including the models of through-body thrusters and control surfaces, manoeuvring tests are performed to forecast the Yangji's performance. Criteria approved by International Maritime Organization (IMO) are then resorted to calculate thresholds for low-speed and high-speed manoeuvres.

\subsection{Turning manoeuvring and its criteria}

In 2002, the following initial turning ability was approved by IMO:

With the application of $10^{\circ}$ rudder angle to port/starboard, as illustrated in Fig. 5, the ship advance should not have traveled more than 2.5 ship 


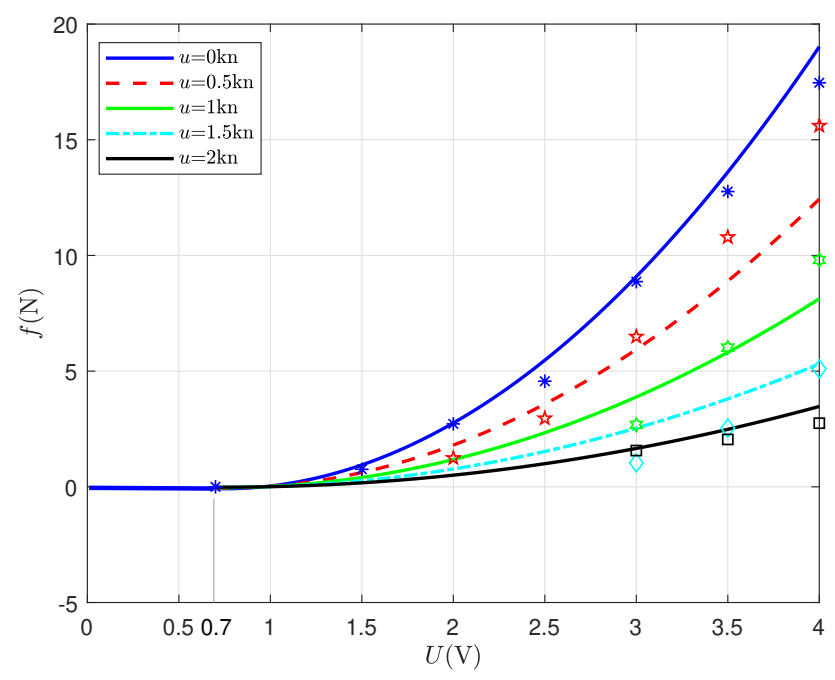

Figure 4: Thrust fitting curve of through-body thrusters

lengths by the time the heading has changed by $10^{\circ}$ from the original heading.

\subsection{Initial turning ability using through-body thrusters}

According to the aforementioned initial turning ability, the breakdown speed of through-body thrusters is first defined:

Definition 1: We can seek certain surge speed $u_{1}$, at which the Yangji's heading changes by $10^{\circ}$ from the original state, the advance is precisely equal to 2.5 times the length of this AUV with the application of $2.9 \mathrm{~V}$ control voltage. In this paper, this surge speed $u_{1}$ is defined as the breakdown speed of through-body thrusters.

Remark 2. For the Yangji AUV, the maximum inputs of through-body thrusters and control surfaces are set to $4 \mathrm{~V}$ and $35^{\circ}$, respectively. Hence, we choose $2.9 \mathrm{~V}$ control voltage in terms of $4 \times(1-10 / 35)$.

In order to seek $u_{1}$, the manoeuvring data including turning torque, time and advance are first collected by choosing a series of surge speeds as control variables of complete model-based manoeuvring simulation and are listed in Table 3. It intuitively shows the effective torques generated by through-body thrusters decrease monotonously with the increase of the surge speed; hence, the corresponding advances increase reversely. Table 3 only lists data of surge 


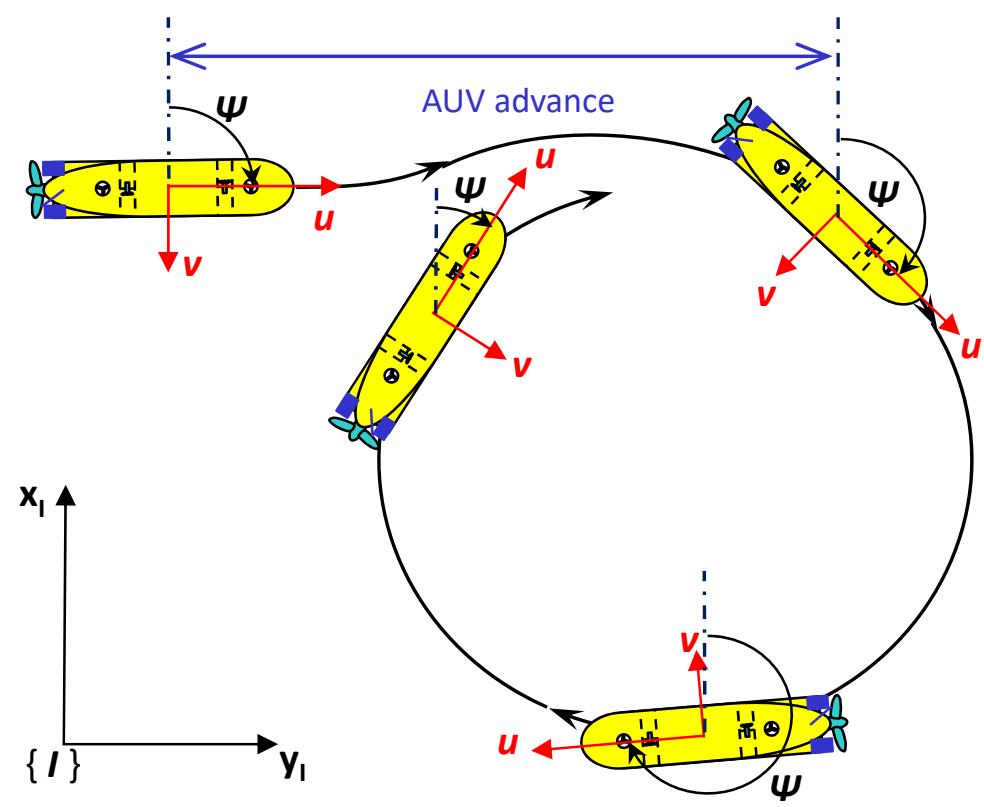

Figure 5: AUV turning manoeuvring and advance definition

speed $u \leq 2 \mathrm{kn}$, because the advance will be obviously larger than 2.5 times the Yangji's length when operating at higher speed.

Subsequently, the interpolated curve of surge distance $d$ with surge speed $u$ is drawn in Fig. 6. The breakdown speed of through-body thrusters can be obtained by locating the intersection of the threshold line and the interpolated cure. From Fig. 6, it can be concluded that the breakdown speed $u_{1}$ of through-body thrusters is $1.39 \mathrm{kn}$, namely $0.714 \mathrm{~m} / \mathrm{s}$. Therefore, through-body thrusters can be seen as enough effective when the Yangji AUV is operated in the low-speed interval of $[0,0.714] \mathrm{m} / \mathrm{s}$; in contrast, the effective thrust of through-body thrusters will undergo a significant reduction and through-body thrusters not be used beyond their breakdown speed.

Finally, the interpolated curve of turning time $t$ with surge speed $u$ is shown in Fig. 7. As the surge speed increases, the turning time will become long. From Fig. 7, we can obtain that the total turning time of through-body thrusters is $14.6 \mathrm{~s}$ at the breakdown speed.

\subsection{Initial turning ability using control surfaces}

As the advances are the same at different speeds when the AUV is operated by setting the same angle of control surfaces [11], the aforementioned 
Table 3: AUV advance using through-body thrusters

\begin{tabular}{ccccc}
\hline Speed $/ \mathrm{kn}$ & Voltage/V & Torque/N·m & Advance/m & Time/s \\
\hline 0.0 & 2.9 & 26.277 & 0 & 6.5 \\
0.25 & 2.9 & 21.246 & 0.96 & 7.5 \\
0.5 & 2.9 & 17.178 & 2.20 & 8.6 \\
0.75 & 2.9 & 13.889 & 3.84 & 10.0 \\
1.0 & 2.9 & 11.229 & 5.99 & 11.7 \\
1.25 & 2.9 & 9.079 & 8.65 & 13.5 \\
1.5 & 2.9 & 7.341 & 11.92 & 15.5 \\
1.75 & 2.9 & 5.935 & 15.70 & 17.5 \\
2.0 & 2.9 & 4.799 & 20.05 & 19.6 \\
\hline
\end{tabular}

initial turning ability cannot be directly adopted. However, as the surge speed increases, the corresponding turning time will decrease. In this paper, the critical speed of control surfaces is defined as follows:

Definition 2: We can also seek certain surge speed $u_{2}$, at which the Yangji's heading changes by $10^{\circ}$ from the original state with the application of $10^{\circ}$ rudder angle, the total time is precisely equal to the time corresponding to the breakdown speed of through-body thrusters. In this paper, this surge speed $u_{2}$ is defined as the critical speed of control surfaces.

The manoeuvring data using control surfaces are listed in Table 4, which shows the effective torque due to control surfaces increases rapidly and the total turning time decreases with the increase of the surge speed; hence, the efficiency of control surfaces will increase with the surge speed.

Table 4: AUV turning time using control surface

\begin{tabular}{cccc}
\hline Speed $/ \mathrm{kn}$ & Rudder $^{\circ}$ & Torque/N·m & Time/s \\
\hline 0.0 & 10 & 0 & $\infty$ \\
0.5 & 10 & 1.4007 & 50.5 \\
1.0 & 10 & 5.6027 & 26.0 \\
1.5 & 10 & 12.6060 & 17.8 \\
2.0 & 10 & 22.4107 & 13.8 \\
3.0 & 10 & 50.4241 & 9.6 \\
4.0 & 10 & 89.6429 & 7.6 \\
\hline
\end{tabular}

Based on the data in Table 4, the interpolated curve of the total turning 


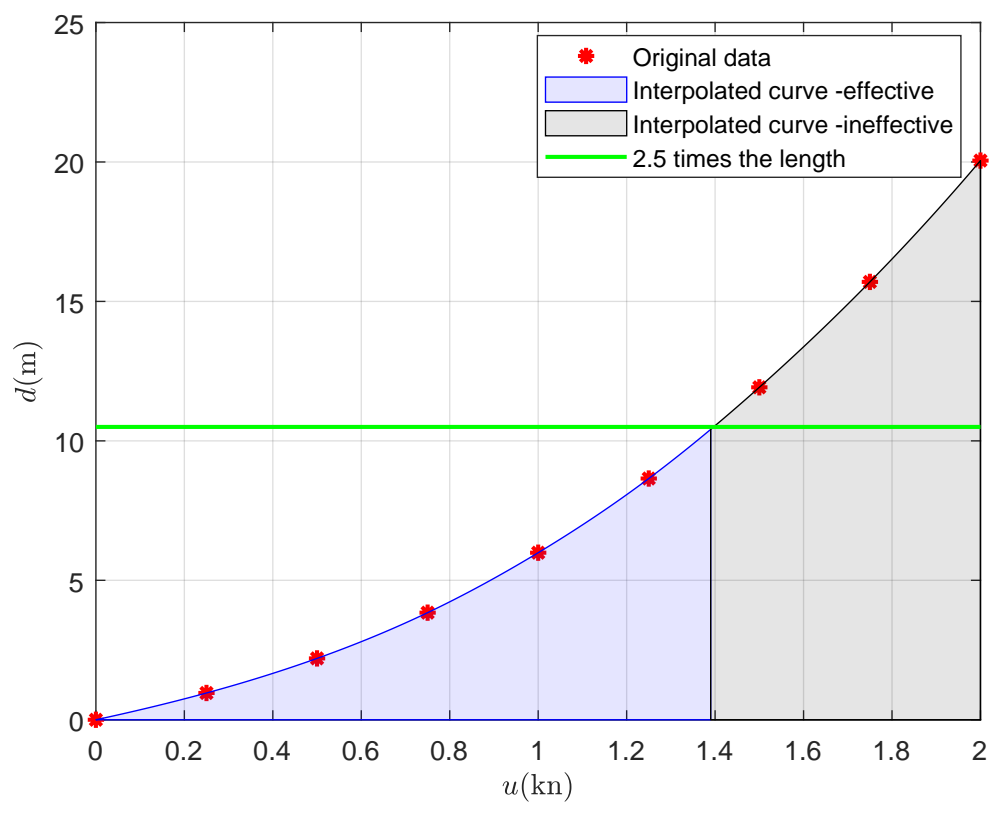

Figure 6: Yangji's Advance against surge speed using through-body thrusters

time $t$ with surge speed $u$ using control surfaces can be illustrated in Fig. 8. Then the critical speed $u_{2}$ of control surfaces is found and equal to $1.88 \mathrm{kn}$, namely $0.966 \mathrm{~m} / \mathrm{s}$. Therefore, the effective torque of control surfaces will be competent for operation at high speed of $[0.966,3.084] \mathrm{m} / \mathrm{s}$, where $3.084 \mathrm{~m} / \mathrm{s}$ is the maximum surge speed of the Yangji AUV.

\subsection{Actuation evaluation}

For the Yangji AUV through-body thrusters should be used in low-speed interval of $[0,0.714] \mathrm{m} / \mathrm{s}$, control surfaces work in high-speed interval of $[0.966,3.084] \mathrm{m} / \mathrm{s}$, and in medium-speed interval of $[0.714,0.966] \mathrm{m} / \mathrm{s}$, the combination of through-body thrusters and control surfaces may be a feasible solution. As the combination of bow and stern horizontal through-body thrusters can generate sway force and yaw moment, the low-speed operation corresponding to the fully-actuated mode; while there is only yaw moment acting on the Yangji AUV using control surfaces, hence at the moment the AUV is under-actuated in sway. Obviously, the Yangji AUV is over-actuated in yaw when both through-body thrusters and control surfaces are used simultaneously in medium-speed interval. The corresponding actuators and 


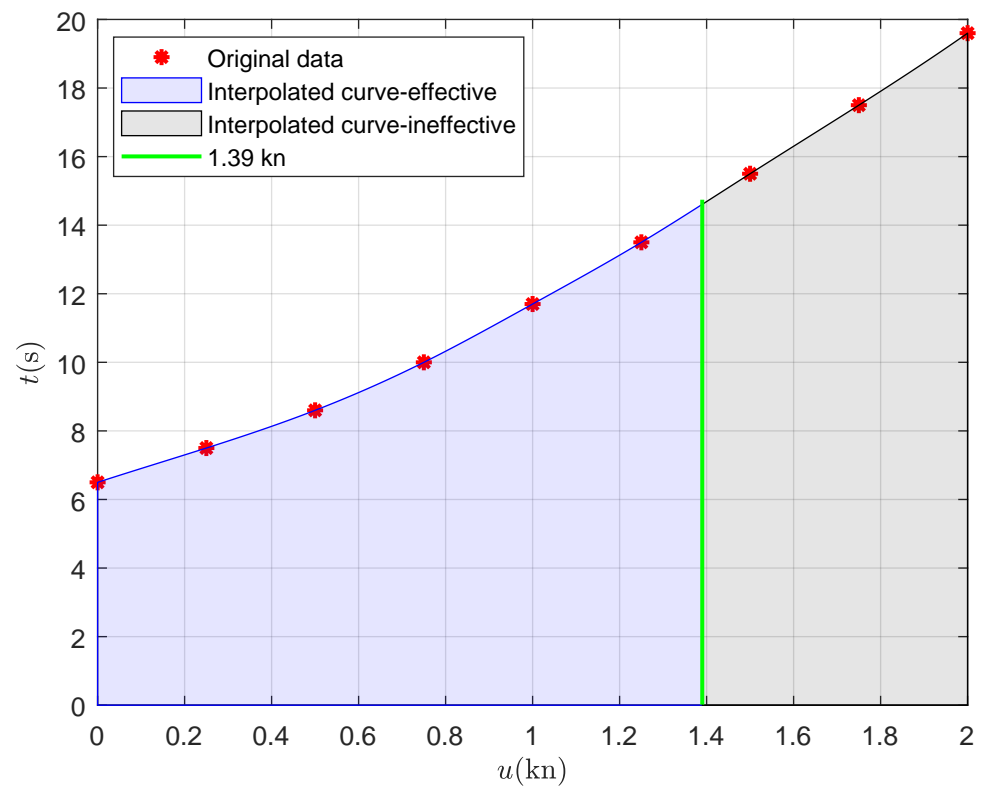

Figure 7: Yangji's turning time against surge speed using through-body thrusters

configuration mode through the entire AUV speed profile are concluded in Table 5.

Table 5: Actuation configuration of the Yangji AUV

\begin{tabular}{cccc}
\hline Mode & Actuator & Speed profile & Speed interval \\
\hline Fully-actuation & Through-body thrusters & Low speed & {$[0,0.714] \mathrm{m} / \mathrm{s}$} \\
Over-actuation & Both & Medium speed & {$[0.714,0.966] \mathrm{m} / \mathrm{s}$} \\
Under-actuation & Control surfaces & High speed & {$[0.966,3.084] \mathrm{m} / \mathrm{s}$} \\
\hline
\end{tabular}

In order to smooth the medium-speed interval, a smooth switching law is designed to manage the contribution of each set of actuators according to the surge speed, as follows:

$$
\left\{\begin{array}{l}
f_{\text {surface }}(u)=0.5 \tanh \left(25\left(u-\frac{u_{1}+u_{2}}{2}\right)\right)+0.5 \\
f_{\text {thruster }}(u)=1-f_{\text {surface }}
\end{array}\right.
$$

where $f_{\text {surface }}$ represents the control surface weighting function and $f_{\text {thruster }}$ is the through-body thruster weighting function. 


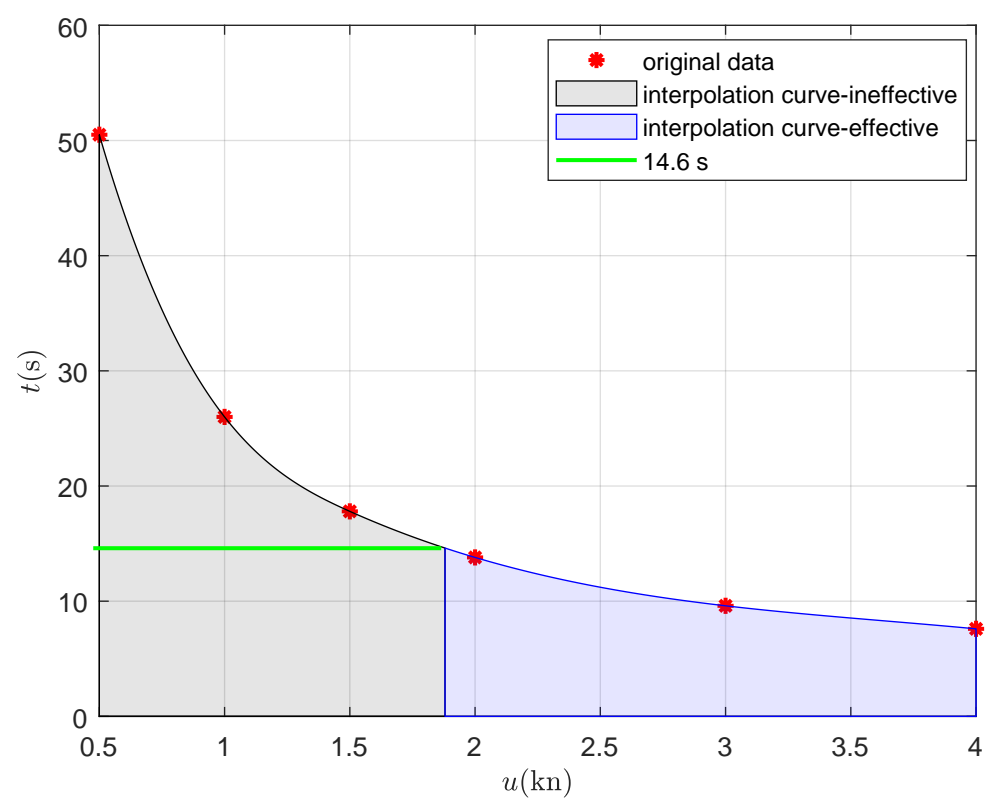

Figure 8: Turning time against surge speed using control surfaces

The final weighting functions for through-body thrusters and control surfaces that are overlapping over the intermediate transition interval are shown in Fig. 9. As the surge speed $u$ increases, thruster weight, $f_{\text {thruster }}$, decreases from 1 to 0 ; therefore, the use of through-body thrusters is entirely removed in the high-speed interval. By contrast, the reliance on control surfaces gradually increases with surge speed $u$. Applying (9) to two kinds of actuators leads to the integrated yaw moment, as shown in Fig. 10, where the output using the designed smooth switching law is smooth through the medium speed profile, compared to the line superposition.

Remark 3. Table 5 and Fig. 9 guide the Yangji AUV to smoothly cross its entire non-zero speed envelope. Then, an unified tracking control for the Yangji AUV from hover to flight can be completed by using the proposed method in $[29,30,31]$. Although the speed intervals are only suitable for the Yangji AUV, the solution steps including dynamics modelling, turning manoeuvring test and data analysis apply to the kind of AUVs equipped with through-body thrusters and control surfaces. Hence, it will provide support for controlling the vehicle through all the stages from low-speed to high-speed maneuvering. 


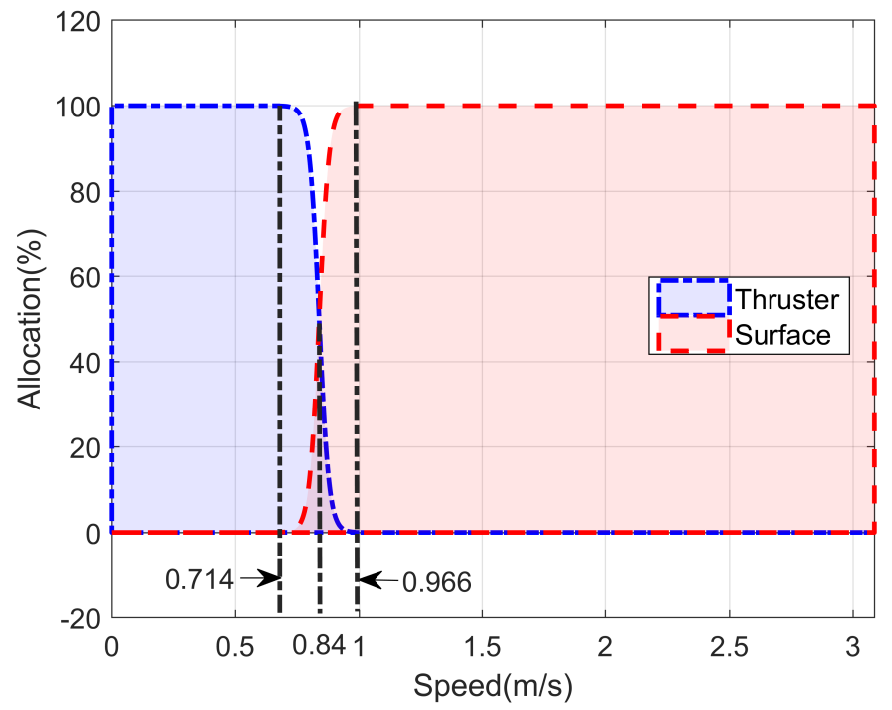

Figure 9: Smooth switching law through the entire speed profile

\section{Conclusions}

In this paper, the accurate speed intervals of low-speed and high-speed manoeuvres are quantitatively analyzed. To the best knowledge of the authors, this is the first study evaluating speed intervals for an AUV. The results show that low-speed and high-speed intervals are not separated by a point, in contract, a medium-speed interval can be introduced where both through-body thrusters and control surfaces are used simultaneously. To smooth the contribution of two types of actuators, a smooth switching law is designed through the entire AUV speed profile. Future work could involve the unified control of the Yangji AUV from hover to flight.

\section{Acknowledgement}

This work was supported in part by the National Natural Science Foundation of China under Grants 51579111, 51209100 and 51909161, in part by the Shenzhen Science and Technology Plan Project under Grant JCYJ201704I311305468, in part by the International Exchanges of the UK Royal Society under Grant IE161588, in part by the Shanghai Sailing Program under Grant 19YF1424100, and in part by the Startup Fund for Youngman Research at Shanghai Jiao Tong University under Grant 19X100040001. 


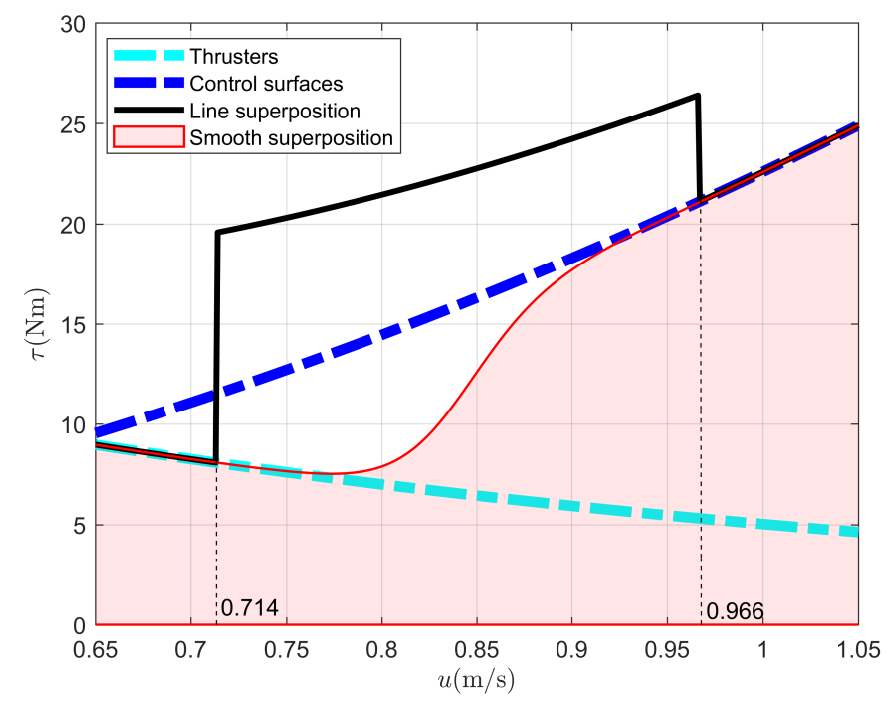

Figure 10: Smooth switching law through the entire speed profile

[1] S. Cohan, Trends in rov development, Marine Technology Society Journal 42 (1) (2008) 38-43.

[2] F. Zhang, G. Marani, R. N. Smith, H. T. Choi, Future trends in marine robotics, IEEE Robotics \& Automation Magazine 22 (1) (2015) 14-122.

[3] D. L. Rudnick, S. T. Cole, On sampling the ocean using underwater gliders, Journal of Geophysical Research 116 (C8) (2011) C08010.

[4] A. Philips, L. Steenson, E. Rogers, S. Turnock, C. Harris, M. Furlong, Delphin2: An over actuated autonomous underwater vehicle for manoeuvring research, Transactions of the Royal Institution of Naval Architects (Part A)-International Journal of Maritime Engineering 155 (A4) (2013) 171-180.

[5] X. Xiang, C. Yu, Z. Niu, Q. Zhang, Subsea cable tracking by autonomous underwater vehicle with magnetic sensing guidance, Sensors 16 (8) (2016) 1335.

[6] C. Yu, X. Xiang, L. Lapierre, Q. Zhang, Robust magnetic tracking of subsea cable by auv in the presence of sensor noise and ocean currents, IEEE Journal of Oceanic Engineering 43 (2) (2018) 311-322. 
[7] L. V. Steenson, A. B. Phillips, E. Rogers, M. E. Furlong, S. R. Turnock, Control of an auv from thruster actuated hover to control surface actuated flight, in: Proceedings of the Specialists Meeting AVT-189/RSM-028 Assessment of Stability and Control Prediction Methods for NATO Air \& Sea Vehicles, Southampton, UK, 2011, pp. 1-13.

[8] M. Pfingsthorn, R. Rathnam, T. Luczynski, A. Birk, Full 3d navigation correction using low frequency visual tracking with a stereo camera, in: OCEANS 2016 - Shanghai, 2016, pp. 1-6. doi:10.1109/OCEANSAP.2016.7485520.

[9] X. Xiang, C. Yu, Q. Zhang, On intelligent risk analysis and critical decision of underwater robotic vehicle, Ocean Engineering 140 (2017) 453-465.

[10] M. Carreras, J. D. Hernández, E. Vidal, N. Palomeras, D. Ribas, P. Ridao, Sparus ii auv - a hovering vehicle for seabed inspection, IEEE Journal of Oceanic Engineering 43 (2) (2018) 344-355.

[11] X. Liang, Y. Li, Z. Peng, J. Zhang, Nonlinear dynamics modeling and performance prediction for underactuated auv with fins, Nonlinear Dynamics 84 (1) (2016) 237-249.

[12] L. Chen, R. Cui, C. Yang, W. Yan, Adaptive neural network control of underactuated surface vessels with guaranteed transient performance: Theory and experimental results, IEEE Transactions on Industrial Electronics (2019) 1-1doi:10.1109/TIE.2019.2914631.

[13] Z. Zheng, C. Jin, M. Zhu, K. Sun, Trajectory tracking control for a marine surface vessel with asymmetric saturation actuators, Robotics and Autonomous Systems 97 (2017) 83-91.

[14] X. Xiang, C. Yu, L. Lapierre, J. Zhang, Q. Zhang, Survey on fuzzy-logicbased guidance and control of marine surface vehicles and underwater vehicles, International Journal of Fuzzy Systems 20 (2) (2018) 572-586.

[15] R. Cui, L. Chen, C. Yang, M. Chen, Extended state observer-based integral sliding mode control for an underwater robot with unknown disturbances and uncertain nonlinearities, IEEE Transactions on Industrial Electronics 64 (8) (2017) 6785-6795. 
[16] Z. Peng, J. Wang, D. Wang, Distributed maneuvering of autonomous surface vehicles based on neurodynamic optimization and fuzzy approximation, IEEE Transactions on Control Systems Technology 26 (3) (2018) $1083-1090$.

[17] N. Wang, S.-F. Su, J. Yin, Z. Zheng, M. J. Er, Global asymptotic modelfree trajectory-independent tracking control of an uncertain marine vehicle: an adaptive universe-based fuzzy control approach, IEEE Transactions on Fuzzy Systems 26 (3) (2018) 1613-1625.

[18] L. Steenson, A. Phillips, E. Rogers, M. Furlong, S. Turnock, The performance of vertical tunnel thrusters on an autonomous underwater vehicle operating near the free surface in waves, in: Proceedings of the Second International Symposium on Marine Propulsors, Hamburg, Germany, 2011, pp. 499-506.

[19] J.-M. Godhavn, T. I. Fossen, S. P. Berge, Non-linear and adaptive backstepping designs for tracking control of ships, International Journal of Adaptive Control and Signal Processing 12 (8) (1998) 649-670.

[20] A. Palmer, G. E. Hearn, P. Stevenson, Modelling tunnel thrusters for autonomous underwater vehicles, in: Proceedings of the 2nd IFAC Workshop on Navigation, Guidance and Control of Underwater Vehicles, Killaloe, Ireland, 2008, pp. 91-96.

[21] K. Tanakitkorn, P. A. Wilson, S. R. Turnock, A. B. Phillips, Depth control for an over-actuated, hover-capable autonomous underwater vehicle with experimental verification, Mechatronics 41 (2017) 67-81.

[22] G. N. Roberts, R. Sutton, Advances in unmanned marine vehicles, The Institution of Electrical Engineers, London, UK, 2006.

[23] T. I. Fossen, Guidance and control of ocean vehicles, New York: Wiley, 1994.

[24] K. Tanakitkorn, Guidance, control and path planning for autonomous underwater vehicles, Ph.D. thesis, University of Southampton (2017).

[25] R. E. D. Bishop, A. G. Parkinson, On the planar motion mechanism used in ship model testing, Philosophical Transactions of the Royal Society 
A: Mathematical, Physical \& Engineering Sciences 266 (1171) (1970) 35-61.

[26] J. E. da Silva, B. Terra, R. Martins, J. B. de Sousa, Modeling and simulation of the lauv autonomous underwater vehicle, in: Proceedings of the 13th IEEE IFAC International Conference on Methods and Models in Automation and Robotics, Szczecin, Poland Szczecin, Poland, 2007, pp. $713-718$.

[27] S. L. Toxopeus, Deriving mathematical manoeuvring models for bare ship hulls using viscous flow calculations, Journal of Marine Science and Technology 14 (1) (2009) 30-38.

[28] F. Xu, Z.-J. Zou, J.-C. Yin, J. Cao, Identification modeling of underwater vehicles' nonlinear dynamics based on support vector machines, Ocean Engineering 67 (2013) 68-76.

[29] X. Xiang, L. Lapierre, B. Jouvencel, Smooth transition of auv motion control: From fully-actuated to under-actuated configuration, Robotics and Autonomous Systems 67 (2015) 14-22.

[30] M. Breivik, T. I. Fossen, A unified control concept for autonomous underwater vehicles, in: Proceedings of the 2006 American Control Conference, 2006, pp. 4920-4926.

[31] K. Tanakitkorn, P. A. Wilson, S. R. Turnock, A. B. Phillips, Sliding mode heading control of an overactuated, hover-capable autonomous underwater vehicle with experimental verification, Journal of Field Robotics 35 (3) (2018) 396-415. 